\title{
Research on Coupled Dynamic Model of Tracked Vehicles and Its Solving Method
}

\author{
Yuliang Li and Chong Tang \\ School of Mechanical Engineering, Zhejiang University, Hangzhou 310027, China \\ Correspondence should be addressed to Yuliang Li; lyl_zw@zju.edu.cn
}

Received 11 October 2014; Revised 6 February 2015; Accepted 6 February 2015

Academic Editor: Ricardo Aguilar-López

Copyright (C) 2015 Y. Li and C. Tang. This is an open access article distributed under the Creative Commons Attribution License, which permits unrestricted use, distribution, and reproduction in any medium, provided the original work is properly cited.

\begin{abstract}
In order to conveniently analyze the dynamic performance of tracked vehicles, mathematic models are established based on the actual structure of vehicles and terrain mechanics when they are moving on the soft random terrain. A discrete method is adopted to solve the coupled equations to calculate the acceleration of the vehicle's mass center and tractive force of driving sprocket. Computation results output by the model presented in this paper are compared with results given by the model, which has the same parameters, built in the multi-body dynamic software. It shows that the steady state calculation results are basically consistent, while the model presented in this paper is more convenient to be used in the optimization of structure parameters of tracked vehicles.
\end{abstract}

\section{Introduction}

Tracked vehicles are widely used in the field of military, agriculture, and construction industries. They are applied on the ground that has a low carrying capacity or wheeled vehicles cannot go through (swamp, snowfield, etc.) or are used for obstacle-crossing and climbing, and so forth because the tracks can be treated as self-carried road to increase the vehicle's passing ability. Nowadays, there are lots of modeling methods about tracked vehicles which primarily treat the tracks as flexible [1-5] or rigid ones [6-9].

$\mathrm{Ma}$ and Perkins employed a nonlinear finite element method to represent the track segment contacting with the terrain $[1,2]$; Park et al. divided the track system contacting with terrain into a finite number of small elements and used structural analysis and the numerical iterative method to calculate the drawbar pull force [3]; Ma treated the tracks as flexible tracks and exerted the excitation directly on the road wheels to reduce the complexity of the model [4]; Han et al. regarded tracks as flexible ones and used springs to replace tracks between road wheels in order to build the ride comfort model of the tracked vehicle [5].

The assumption of flexible tracks cannot fully reflect the dynamic performance of tracks. Rigid models are adopted by more researchers. Merhof and Hackbarth illustrated the actual structure of the tracks and set up their dynamic equations [6]; Rubinstein and Hitron described each track's positions, movements, and the interaction with terrain by coordinate transformation in three-dimensional space [7]; Ma et al. regarded the tracks and the road wheels as a whole and analyzed each part in the track system [8]; Hu took heavy tracked vehicle as a research object and divided the track system into 4 parts, namely, the tracks in contact with terrain, the tracks in contact with front idler, the tracks in contact with driving sprocket, and the tracks between front idler and driving sprocket [9].

Although the assumption of rigid tracks is approaching the truth, the effects of structure parameters on the performance are difficult to analyze, and generally the model cannot be used in the structural optimization. Therefore, this paper presents a simplified dynamic model based on the assumption of rigid tracks. A discrete method is used to solve the coupled equations. The computation results, namely, the acceleration of the vehicle's mass center and the tractive force of driving sprocket, are compared with those obtained from RecurDyn to verify the model's validity.

\section{Dynamic Model of Tracked Vehicles}

2.1. Random Road Excitations. In this paper grade E road surface is taken as the random excitations for the tracked 


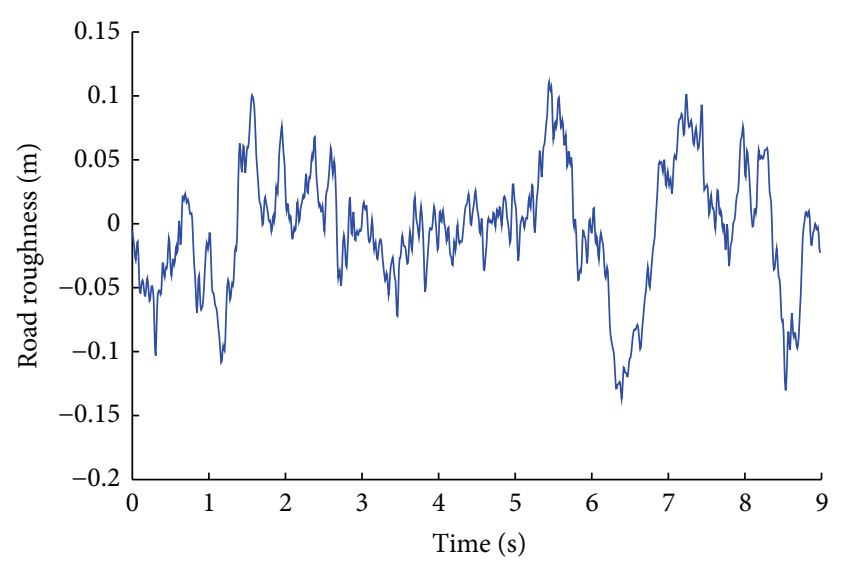

FIgURE 1: Grade E random road surface.

vehicles. The road model is built by making use of power spectral density function in time frequency domain $G_{q}(f)$ [10-12] and the iteration of trigonometric series method [13]; the result is illustrated in Figure 1. The tracked vehicle's highest speed is $60 \mathrm{~km} / \mathrm{h}$, the length of a track is $0.153 \mathrm{~m}$, and so the time that a wheel passes by a track is $0.009 \mathrm{~s}$. Thus the time interval for the discrete sampling of terrain excitation $y(j)$ is $0.009 \mathrm{~s}$ in order to simulate the vehicle run on the road surface.

2.2. Track-Terrain Contact. Currently the most widely used model for analyzing soil sinkage is Bekker's equation [14, 15]:

$$
\sigma=\left(\frac{k_{c}}{b}+k_{\phi}\right) z^{n}
$$

where, $\sigma$ is the pressure, kpa; $n$ is the pressure-sinkage exponent; $k_{c}$ is the soil cohesive coefficient, $\mathrm{kN} / \mathrm{m}^{n+1} ; k_{\phi}$ is the soil internal friction coefficient, $\mathrm{kN} / \mathrm{m}^{n+2} ; b$ is the width of a track, $\mathrm{m} ; z$ is the sinkage, $\mathrm{m}$.

Actually the tracked vehicle will have repeated loads on the same terrain when different wheels of the vehicle pass by it. This phenomenon can be more precisely described by the loading-unloading curve of soil model introduced by Wong [16] than Bekker's equation, as shown in Figure 2.

As it can be seen from Figure 2, OA is the continuous loading curve which can be described by (1); BA is the unloading and repeated loading curve in which the slope can be seen as a constant value $k_{u}$, and the curve can be described by [17]

$$
\sigma= \begin{cases}\left(\frac{k_{c}}{b}+k_{\varphi}\right) z^{n} & \mathrm{OA} \\ \left(\frac{k_{c}}{b}+k_{\varphi}\right) z_{u}{ }^{n}-k_{u}\left(z_{u}-z\right) & \mathrm{BA},\end{cases}
$$

where $z_{u}$ is the maximum sinkage, $\mathrm{m} ; z$ is the sinkage, $\mathrm{m}$.

In order to build and solve the dynamic models of tracked vehicles, some simplifications, which have little effects on the final calculation results, are needed: (1) every $y(j)$ is sequentially exerted on the two pins of a track so that the terrain under the track would be complete and continuous;

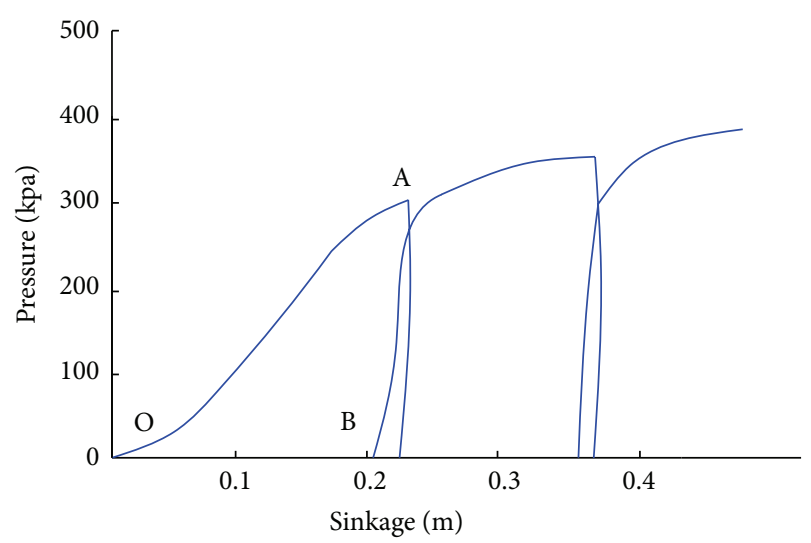

FIGURE 2: Loading-unloading curve of soil [16].

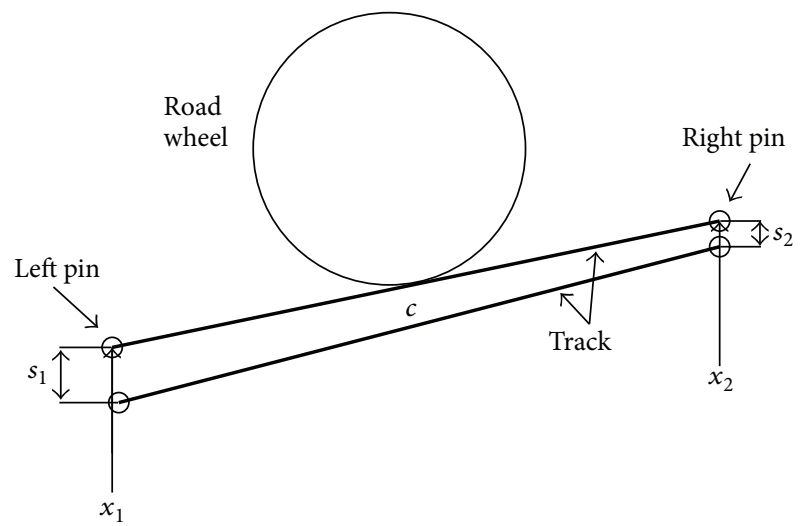

FIGURE 3: Average sinkage, where, $c$ is the midpoint of the track; $x$ is the road excitation; $s$ is the average sinkage under the pins, namely $s=\left(s_{1}+s_{2}\right) / 2$.

this will lay the foundation for the calculation of shear forces acting on tracks by terrain; (2) in reality the angle of track changes when a boogie passes by it. However, since the time is very short, it is reasonable to assume that the track keeps the same angle during this period so that the coupled relationships among wheel, track, and terrain can be decoupled; (3) the average sinkage of both pins of a track after the wheel leaves it is regarded as the sinkage of the track when the wheel is on the middle of it, as shown in Figure 3.

At the first sampling time, terrain excitations on both pins of the track under the first road wheel are original excitations $y(1)$ and $y(0)$. At the second sampling time, the excitations on the track under the first wheel are $y(2)$ and $y(1)$ that has sinkage and springback after the first sampling time and so are the following excitations on the track. For the tracks under the second to the sixth road wheels, the terrain excitations on both pins of them are excitations that have sinkage and springback after the former sampling time. Besides, there are 2 tracks at the right and left sides of one terrain excitation and at least one road wheel has passed by before. Therefore, all of the former sinkage and springback should be taken into consideration; namely, the original excitation should subtract the maximum average sinkage among all the ones and then 


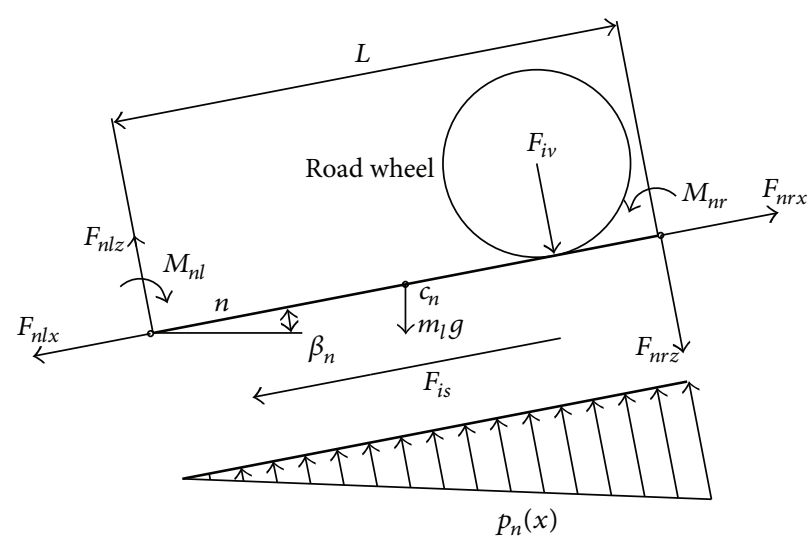

FIGURE 4: Forces and moments acting on a track contacting with a road wheel, where, $n$ and subscript $n$ are the $n$th track, the same as below; subscripts $i, l, r, s, x, z$, and $v$ are the $i$ th road wheel, left pin, right pin, shear force, the direction along the track, the direction vertical to the track, and road wheel's vertical force on the track, the same as below; $c_{n}$ is the midpoint of the $n$th track; $F_{n l x}, F_{n r x}$ is the force components acting on both pins in the direction along the track, $\mathrm{kN} ; F_{n l z}, F_{n r z}$ is force components acting on both pins in the direction vertical to the track, $\mathrm{kN} ; M_{n l}, M_{n r}$ is the moments acting on both pins of the track, $\mathrm{kN} \cdot \mathrm{m} ; F_{i v}$ is the vertical force acting on the track from road wheel, which equals the sum of the weight of a road wheel, inertial force of a road wheel and the suspension's reactive force, $\mathrm{kN} ; F_{i s}$ is the shear force acting on a track from terrain, $\mathrm{kN} ; g$ is the acceleration of gravity, $\mathrm{m} / \mathrm{s}^{2} ; m_{l}$ is the mass of a track, $\mathrm{kg}$; $L$ is the length of a track, $\mathrm{m} ; \beta_{n}$ is the angle of the track, $\operatorname{rad} ; p_{n}(x)$ is the pressure distribution function under a track, kpa.

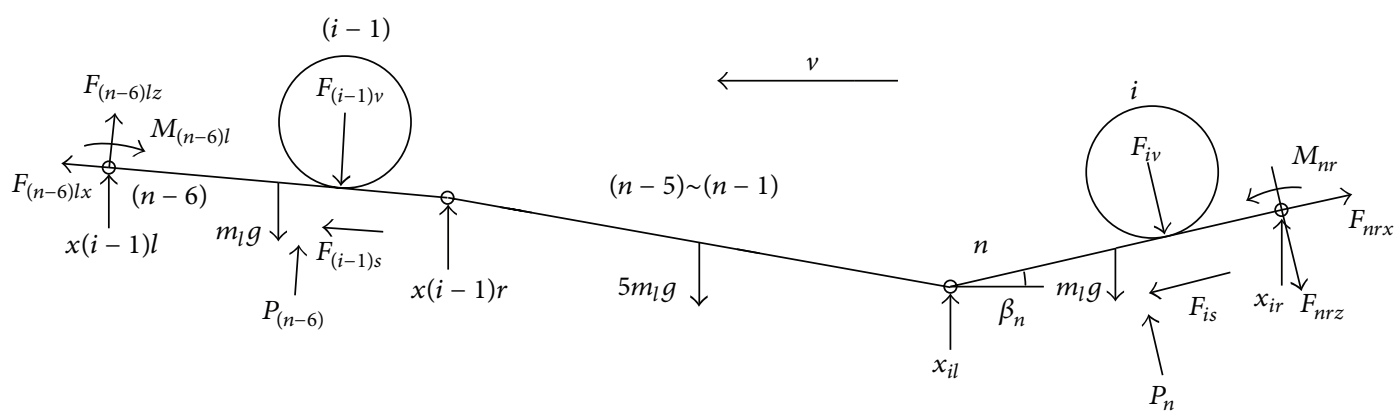

FIGURE 5: Geometric and mechanical relationships between tracks, where $P_{n}$ is the concentrated force obtained by summing the forces distributed along the whole track, $\mathrm{KN}$.

add the springback to form the new excitations acting on the later tracks.

2.3. Mechanical Analysis of Tracks. In this model the inertial force and inertial moment of a track are neglected due to the fact that the mass of a track is very small compared with the whole vehicle. Thus, the forces and moments acting on a track can be illustrated in Figure 4.

In order to compute the shear force acting on a track, the first step is to calculate $F_{n l z}, F_{n r z}$ and $M_{n l}, M_{n r}$. Here, it is supposed that the time period that a road wheel passes a track is $d t$. During this period the angle of a track, vertical and angular acceleration of the vehicle's mass center, the vertical force acting on a track from road wheel $F_{i v}$, and the sinkage of excitation are calculated every $d t$, other physical variables are calculated every $d t / 3$. Therefore, there are 4 calculation points in $d t$ except the midpoint. Figure 5 illustrates this concept.

Firstly, let us analyze the interaction between the first road wheel and the track under it, and the analysis result also applies to other wheels and tracks. It is supposed that the tracked vehicle is initially static on a level terrain. After a $d t$ the angle of the track contacting with the road wheel $(i-1$, $i=2$ ) changes to $\beta_{n}$ because of the excitation from terrain. $F_{n l x}, F_{n l z}$ acting on the track can be obtained from Figure 6:

$$
\begin{aligned}
& F_{n l x}=F_{a}^{\prime} \cos \left(\beta_{a}-\theta_{c}+\beta_{n}\right), \\
& F_{n l z}=F_{a}^{\prime} \sin \left(\beta_{a}-\theta_{c}+\beta_{n}\right) .
\end{aligned}
$$

Two force components of the pretensioning force, namely, $F_{n r x}$ and $F_{n r z}$, are acting on the right pin. Figure 7 illustrates all the forces acting on the track at the first calculation point in the time period $d t$.

The pressure distribution $P_{n}$ acting on the track from terrain is obtained by Figure 7 and the shear force (mentioned in Section 2.4) at the first calculation point can be calculated.

In order to solve the coupled equations, $\beta_{n}, F_{(i-1) v}, F_{n l x}$, $F_{n l z}, P_{n}$, and $F_{(i-1) s}$ are kept unchanged at the next calculation point. At the second calculation point, $F_{n r x}, F_{n r z}$, and $M_{n r}$ 


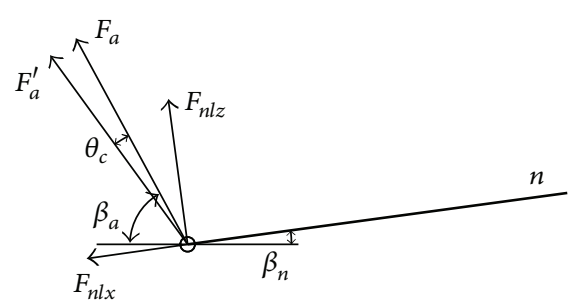

FIGURE 6: Forces acting on the left pin of the track under the first road wheel, where $F_{a}$ is the pretensioning force of tracks, $\mathrm{kN} ; \theta_{c}$ is the rotation angle of the vehicle's mass center, rad; $\beta_{a}$ is the angle between the tracks that link front idler and the level terrain when the vehicle is static, rad. superscript " " "represents the values obtained at the former calculation point, the same as below (see (3) and (4)).

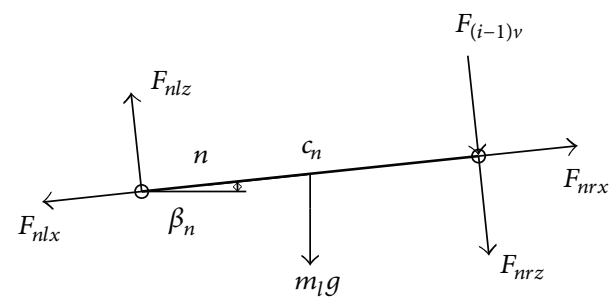

FIGURE 7: Forces acting on a track at the first calculation point in the first time period $d t$.

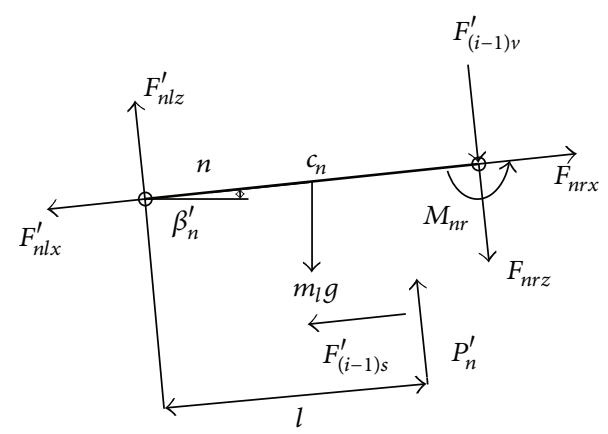

FIGURE 8: Calculation of $F_{n r x}, F_{n r z}$, and $M_{n r}$ at the second point in the time period $d t$.

can be obtained according to equilibrium conditions. For instance, in Figure 8, we can have

$$
\begin{gathered}
F_{n r x}=F_{n l x}^{\prime}+F_{(i-1) s}^{\prime}+m_{l} g \sin \left(\beta_{n}^{\prime}\right), \\
F_{n r z}=F_{n l z}^{\prime}+P_{n}^{\prime}-m_{l} g \cos \left(\beta_{n}^{\prime}\right)-F_{(i-1) v}^{\prime}, \\
M_{n r}=F_{n l z}^{\prime} L-m_{l} g \cos \left(\beta_{n}^{\prime}\right)\left(\frac{L}{2}\right)+P_{n}^{\prime}(L-l) .
\end{gathered}
$$

In Figure 9, $F_{(i-1) v}$ moves $L / 3$ forward, the pressure distribution acting on the track from terrain and the shear force can be calculated by $F_{n r x}, F_{n r z}$, and $M_{n r}$ (5) and the unchanged values of $\beta_{n}, F_{(i-1) v}, F_{n l x}, F_{n l z}, P_{n}$, and $F_{(i-1) s}$. Based on this method, the similar values can be obtained when the road wheel is at the midpoint, $2 L / 3$, and $L$ of the track.

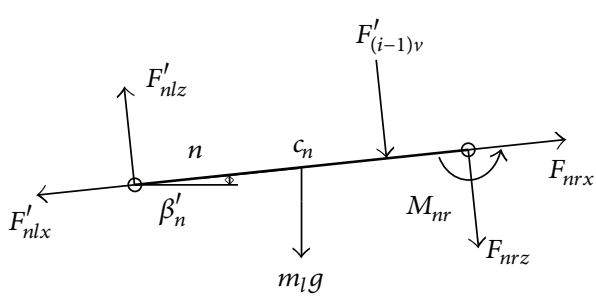

FIGURE 9: Forces acting on the track before the calculation of the shear force at the second calculation point in the time period $d t$.

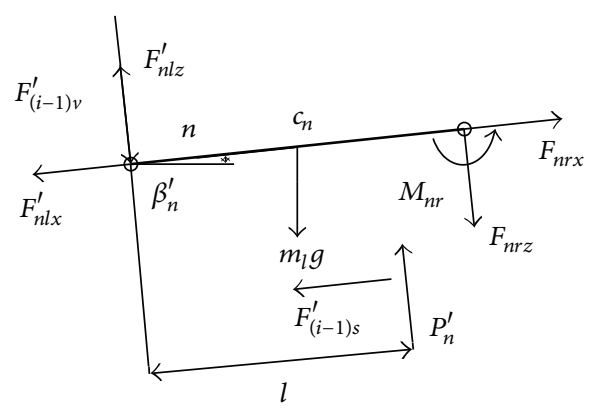

FIGURE 10: The calculation of $F_{n r x}, F_{n r z}$, and $M_{n r}$ at the end of the fourth calculation point in the first $d t$.

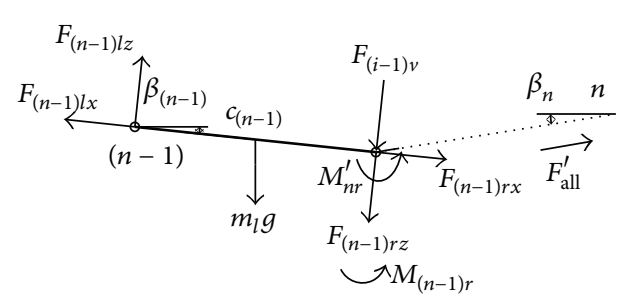

FIGURE 11: Forces acting on the track $(n-1)$ at the first calculation point in the second $d t$.

In the second time period $d t$, road wheel $(i-1)$ moves on to track $(n-1)$. At this junction point the tractive force acting on tracks $(n)$ and $(n-1)$ keeps unchanged. According to Figure 10, after the pressure distribution and shear force are obtained at the fourth calculation point in the first time period $d t, F_{n r x}, F_{n r z}$, and $M_{n r}$ can be calculated as follows:

$$
\begin{gathered}
F_{n r x}=F_{n l x}^{\prime}+m_{l} g \sin \left(\beta_{n}^{\prime}\right)+F_{(i-1) s}^{\prime}, \\
F_{n r z}=F_{n l z}^{\prime}-F_{(i-1) v}^{\prime}-m_{l} g \cos \left(\beta_{n}^{\prime}\right)+P_{n}^{\prime}, \\
M_{n r}=F_{n l z}^{\prime} L-F_{(i-1) v}^{\prime} L-m_{l} g \cos \left(\beta_{n}^{\prime}\right)\left(\frac{L}{2}\right)+P_{n}^{\prime}(L-l) .
\end{gathered}
$$

As illustrated in Figure 11, after the road wheel moves onto track $(n-1), F_{(n-1) r x}, F_{(n-1) r z}$ can be obtained from $F_{\text {all }}^{\prime}$, which 


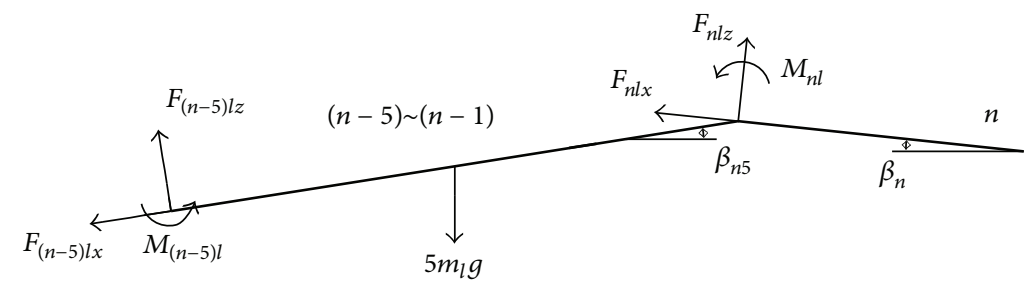

FIGURE 12: Forces acting on the left pin of track $n$ contacting with the second road wheel.

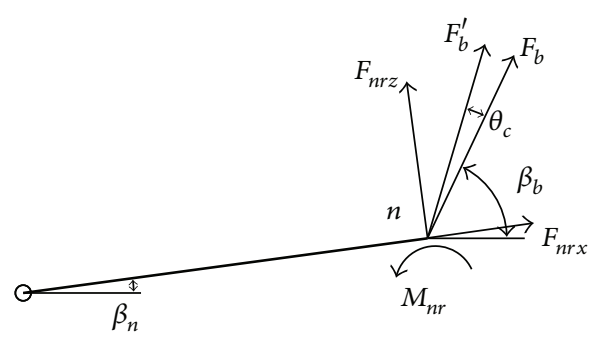

FIGURE 13: Forces acting on a track when the driving sprocket pulls the right pin of it.

is the sum of force components of $F_{n r x}, F_{n r z}$ in the direction along with track $n . M_{(n-1) r}$ equals $M_{n r}^{\prime}$ (obtained by (8)):

$$
\begin{gathered}
F_{(n-1) r x}=F_{\text {all }}^{\prime} \cos \left(\beta_{n}+\beta_{(n-1)}\right), \\
F_{(n-1) r z}=-F_{\text {all }}^{\prime} \sin \left(\beta_{n}+\beta_{(n-1)}\right), \\
M_{(n-1) r}=M_{n r}^{\prime} .
\end{gathered}
$$

To obtain the values at the following calculation points, the method is the same as the calculation points in the first $d t$. When the sixth time period $d t$ comes, random terrain excitation begins to be exerted on the track under the second road wheel because there are 5 tracks between two wheels, as illustrated in Figure 12.

In Figure 12, $F_{(n-5) l x}, F_{(n-5) l z}$ and $M_{(n-5) l}$ act on the same pin as $F_{(n-6) r x}, F_{(n-6) r z}$, and $M_{(n-6) r}$. So they are active and reactive forces (moments). Then $F_{n l x}, F_{n l z}$, and $M_{n l}$ can be computed by the equilibrium condition of track $(n-5)$; namely,

$$
\begin{aligned}
F_{n l x}= & \left(F_{(n-5) l x}+5 m_{l} g \sin \left(\beta_{n 5}\right)\right) \cos \left(\beta_{n 5}+\beta_{n}\right) \\
& +\left(F_{(n-5) l z}-5 m_{l} g \cos \left(\beta_{n 5}\right)\right) \sin \left(\beta_{n 5}+\beta_{n}\right), \\
F_{n l z}= & \left(F_{(n-5) l z}-5 m_{l} g \cos \left(\beta_{n 5}\right)\right) \cos \left(\beta_{n}+\beta_{n 5}\right) \\
& -\left(F_{(n-5) l x}+5 m_{l} g \sin \left(\beta_{n 5}\right)\right) \sin \left(\beta_{n 5}+\beta_{n}\right), \\
& M_{n l}=M_{(n-5) l}-m_{l} g \cos \left(\beta_{n 4}\right)\left(\frac{25 L}{2}\right) .
\end{aligned}
$$

The method to calculate $F_{n r x}, F_{n r z}$, and $M_{n r}$ is the same as the one used to calculate the force or moment values of the track under the first road wheel in the first $d t$. In this way, forces or moments acting on the tracks under and between the road wheels can be obtained.
As illustrated in Figure 13, suppose track $n$ contacts with the sixth road wheel. After calculating $F_{n r x}, F_{n r z}$, and $M_{n r}, F_{b}^{\prime}$ can be obtained by

$$
F_{b}^{\prime}=\frac{F_{n r x}}{\cos \left(\beta_{b}-\beta_{n}+\theta_{c}\right)},
$$

where $F_{b}^{\prime}$ is the driving force given by sprocket, $\mathrm{kN} ; \theta_{c}$ is the rotation angle of the vehicle's mass center, $\operatorname{rad} ; \beta_{b}$ is the angle between the tracks connected with the driving sprocket and the level terrain when the vehicle is static, rad.

2.4. Shear Forces Acting on Tracks. When tracks are pulled from terrain by the driving sprocket, tractive forces will be exerted on them by terrain. On the soft terrain, the tractive force is mainly due to the shear force. Therefore, the tractive force given by the driving sprocket can be obtained by calculating the shear force acting on tracks from terrain [18]. When a road wheel passes by a track in a time period $d t$, the pressure distribution under the track changes according to the wheels' positions, and so do forces and moments on both pins of the track. Figure 14 illustrates the simplification of forces and moments acting on a track at a specific calculation point.

After the simplification, the forces and moments exerted on a track are equal to a force $F$ and a moment $M$. For a rigid and small track, the pressure distribution under the track can be regarded as a linear distribution [19]. There are 3 different situations according to the resultant force $F$ and moment $M$, as illustrated in Figure 15:

$$
\left.\begin{array}{l}
P_{\max } \\
P_{\min }
\end{array}\right\}=\frac{F}{L b}\left(1 \pm \frac{6 e}{L}\right),
$$

where $p_{\max }, p_{\min }$ are the maximum and minimum values of the distribution, $\mathrm{kpa}$; $L$ is the length of a track, $\mathrm{m} ; b$ is the width of a track, $\mathrm{m}$; $e$ is the eccentricity, $e=M / F, \mathrm{~m}$. 

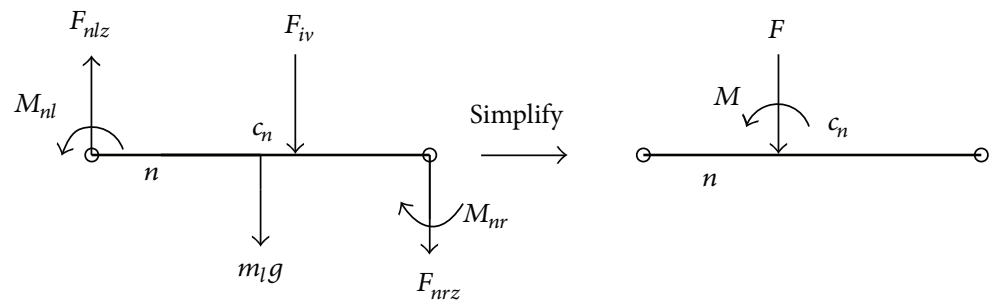

FIGURE 14: Resultant forces of a track.

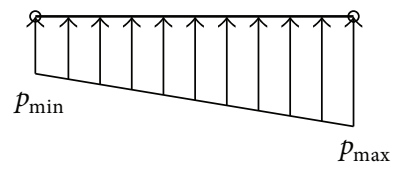

(a)

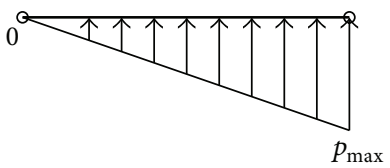

(b)

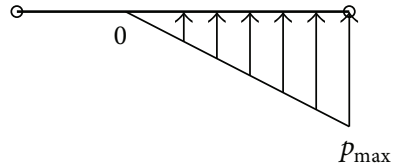

(c)

FIGURE 15: Pressure distributions under a track.

A local coordinate can be placed on a track so that the pressure distribution function can be described as

$$
p(x)=k_{0} x+b_{0}
$$

where $p(x)$ is the pressure distribution function under a track, kpa; $k_{0}$ is the slope; $b_{0}$ is the intercept, $\mathrm{m}$.

Substitute (13) into Janosi shear stress equation [20] (14), shear force acting on tracks from terrain according to different pressure distribution functions can be calculated:

$$
F_{i s}=b \int_{0}^{L}(C+p(x) \tan \varphi)\left(1-e^{-i_{s} L_{1} / K}\right) d x .
$$

Equation (14) can be reduced to

$$
F_{i s}=b\left(1-e^{-i_{s} L_{1} / K}\right)\left(C L+\frac{1}{2} k_{0} L^{2} \tan \varphi+b_{0} L \tan \varphi\right) \text {, }
$$

where $L_{1}$ is the distance that road wheels pass by a track, m; $b$ is the width of a track, $\mathrm{m}$; $C$ is the soil cohesion coefficient, $\mathrm{kN} / \mathrm{m}^{2} ; \varphi$ is the internal friction angle of soil, $\mathrm{rad} ; K$ is the shear modulus deformation of soil, $\mathrm{m} ; i_{s}$ is the sliding rate of vehicle.

2.5. Dynamic Equations of Tracked Vehicles. The following assumptions are made to set up dynamic equations of tracked vehicles: (1) the vehicle is bilaterally symmetrical; (2) excitations exerted on both left and right tracks are exactly the same; (3) road wheels and tracks are rigid. Based on these assumptions, a half vehicle model can be used to build the dynamic model of the vehicle, as illustrated in Figure 16.

(1) Initially the vehicle is static and in equilibrium. So the dynamic equations in $z$ direction are as follows:

$$
\begin{gathered}
m_{c} \ddot{z}_{c}=\sum_{i=1}^{6} F_{p i}+\sum_{k=a, b} F_{k z} \\
F_{a z}=F_{a} \times \sin \left(\beta_{a}+\theta_{c}\right), \quad F_{b z}=F_{b} \times \sin \left(\beta_{b}-\theta_{c}\right),
\end{gathered}
$$

where $m_{c}$ is the mass of half vehicle, $\mathrm{kg} ; z_{c}$ is the displacement of mass center in $z$ direction, $\mathrm{m} ; F_{p i}$ is the forces acting on vehicle' $\mathrm{s}$ body from the suspension system, $\mathrm{kN} ; F_{k},(k=$ $a, b)$ is the forces acting on front idler and driving sprocket of tracks, $\mathrm{kN} ; F_{k z},(k=a, b)$ is the force components in $z$ direction acting on front idler and driving sprocket of tracks, $\mathrm{kN} ; \beta_{k},(k=a, b)$ is the angle between the tracks connecting the driving sprocket and the level road when the vehicle is static, $\mathrm{rad} ; \theta_{c}$ is the rotation angle of the mass center of the vehicle, rad.

(2) Pitching movement equations for the vehicle body,

$$
\begin{aligned}
J_{c} \ddot{\theta}_{c}= & \sum_{i=1}^{6} F_{p i}\left(l_{i} \cos \theta_{c}-h_{i} \sin \theta_{c}\right) \\
& +\sum_{k=a, b} F_{k x}\left(l_{k} \sin \theta_{c}+h_{k} \cos \theta_{c}\right) \\
& +\sum_{k=a, b} F_{k z}\left(l_{k} \cos \theta_{c}-h_{k} \sin \theta_{c}\right),
\end{aligned}
$$

where $J_{c}$ is the inertial moment when the vehicle rotates around the mass center, $\mathrm{kg} \cdot \mathrm{m}^{2} ; F_{k x}(k=a, b)$ is the force components in $x$ direction acting on front idler and driving sprocket, $\mathrm{kN} ; l_{i}, h_{i}(i=1 \sim 6)$ is the horizontal and vertical distances of road wheels' mass center from vehicle's mass center, $l_{i}(i=1,2,3)$ are positive, $l_{i}(i=4,5,6)$ are negative; $l_{k}, h_{k}(k=a, b)$ is the horizontal and vertical distances of front idler's and driving sprocket's mass center from vehicle's mass center, $\mathrm{m} ; l_{a}$ is positive, $l_{b}$ is negative.

(3) Forces exerted by suspension system,

$$
F_{p i}=c \dot{s}_{i}+k s_{i} \text {. }
$$

Since the mass center of the tracked vehicle rotates slightly when it is moving on the grade E random road surface, so

$$
s_{i}=x_{i r}-z_{c}-l_{i} \sin \theta_{c} \approx x_{i r}-z_{c}-l_{i} \theta_{c},
$$

where $c$ is the damping coefficient, $\mathrm{kN} /(\mathrm{m} / \mathrm{s}) ; k$ is the spring coefficient, $\mathrm{kN} / \mathrm{m} ; x_{i r}$ is the excitations exerted on a track's right pin, $\mathrm{m} ; s_{i}$ is the stroke of the suspension system, $\mathrm{m}$. 


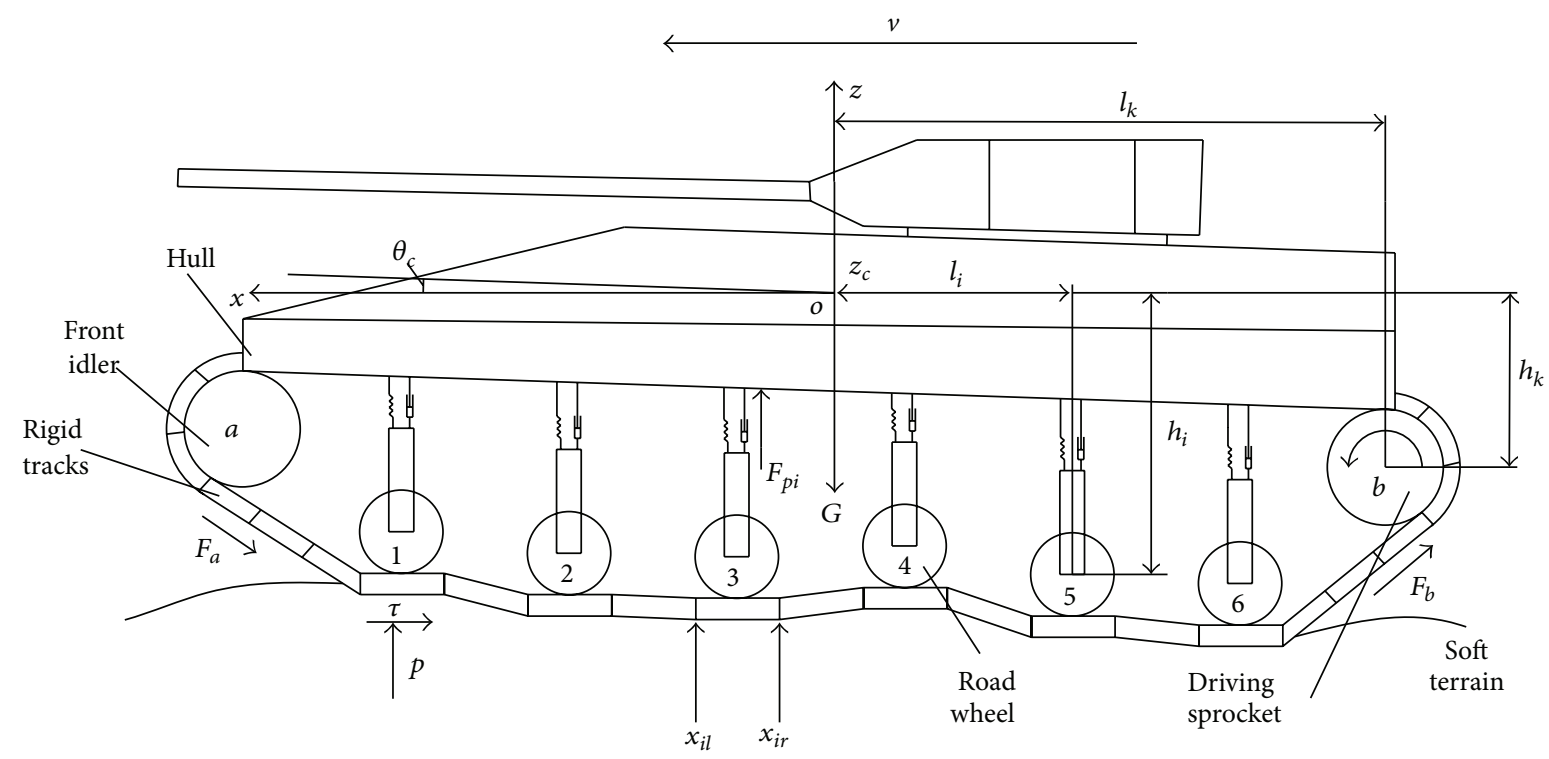

FIGURE 16: Half vehicle model.

(4) The vertical force acting on the track from road wheel, $F_{i v}$,

$$
F_{i v}=\left(F_{p i}+\frac{m_{c}}{6}+m_{z} g-m_{z} a_{i}\right) \times \cos \beta_{n}, \quad(i=1 \sim 6),
$$

where $m_{z}$ is the mass of a road wheel, $\mathrm{kg} ; g$ is the acceleration of gravity, $\mathrm{m} / \mathrm{s}^{2} ; a_{i}$ is the road wheel's acceleration, $\mathrm{m} / \mathrm{s}^{2} ; \beta_{n}$ is the angle of track $n$, rad.

\section{Decoupling of Dynamic Model}

3.1. Coupled Dynamic Model. There exist couplings in the dynamic model of tracked vehicles: (1) if the shear force under a track is to be calculated, it is necessary to know the forces and moments acting on both pins of the track and the vertical force acting on the track by road wheels. But in order to get these forces and moments, the shear force must be first calculated, as mentioned in Section 2.3; (2) in order to solve (16)-(18), the shear force and displacements of springs must be known. But, as described in (1), the shear force should be obtained through the vertical force acting on the track from road wheel and so are the displacements of springs. Obviously, the vertical force is related to the vibration and pitching movement of the tracked vehicle's mass center, which means (16)-(18) are required to calculate the force. The coupling relationships among them are illustrated in Figure 17.

3.2. Model Decoupling Process. The process is shown in Figure 18. Discretization is adopted to solve the coupled dynamic model. A very short sampling time is taken to precisely calculate the derivation and integral of equations or to obtain all the parameters that are needed to calculate other ones, and then substitute the parameters obtained at the former calculation point into the equations to get the

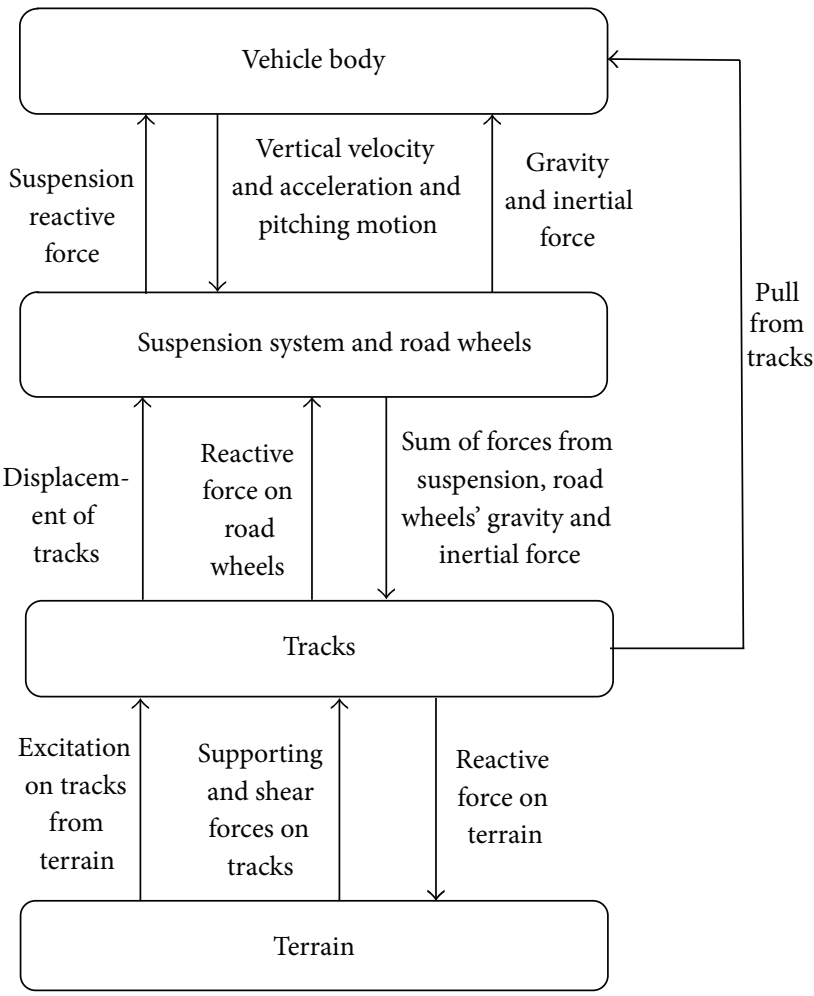

FIGURE 17: Coupling relationships between tracked vehicle and terrain.

parameters for the next calculation point. In the decoupling process, the pressure distribution and the shear force are calculated every $d t / 3$ when a road wheel passes by a track. The vertical and angular velocities of the mass center and so forth are calculated every $d t$, in which $F_{b}$, calculated at the fourth calculation point in a $d t$, must be known beforehand. 


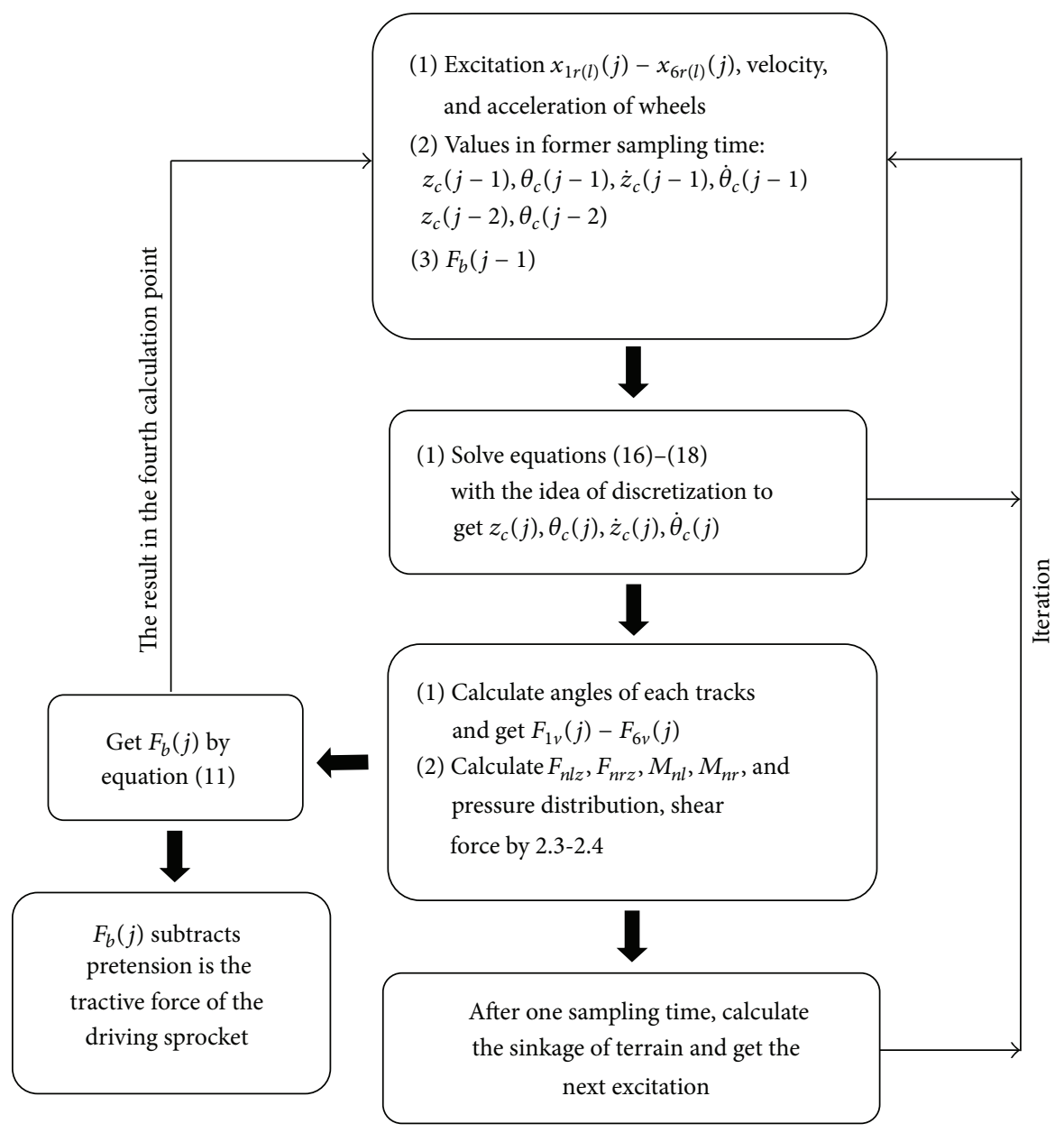

FIGURE 18: Decoupling process.

When the road wheel is at the midpoint of a track, the method introduced in Section 2.3, namely, the calculation equations for the pressure distribution at every calculation point, can be adopted to get the pressure distribution values, and thus the average sinkage of the track can be obtained.

\section{Case Study}

The main parameters of a tracked vehicle are shown in Table 1. The slipping rate of the vehicle $i_{s}=0.025$, the sampling time period $d t=0.009 \mathrm{~s}$. Parameters of the soil, that is, sandy loam, are $k_{c}=52.53 \mathrm{kN} / \mathrm{m}^{n+1}, k_{\varphi}=1127.97 \mathrm{kN} / \mathrm{m}^{n+2}, C=$ $4.83 \mathrm{kN} / \mathrm{m}^{2}, \varphi=0.349 \mathrm{rad}, K=0.025 \mathrm{~m}$, and $n=0.9$, and the maximum sinkage $=0.2 \mathrm{~m}$.

A virtual prototype model of the tracked vehicle was built using the module "Track-HM" in RecurDyn to test the accuracy of calculation, as illustrated in Figure 19. The parameters used to build the virtual prototype are the same as the ones used in this paper, as shown in Table 1. Considering it needs some time for the virtual prototype model to accelerate

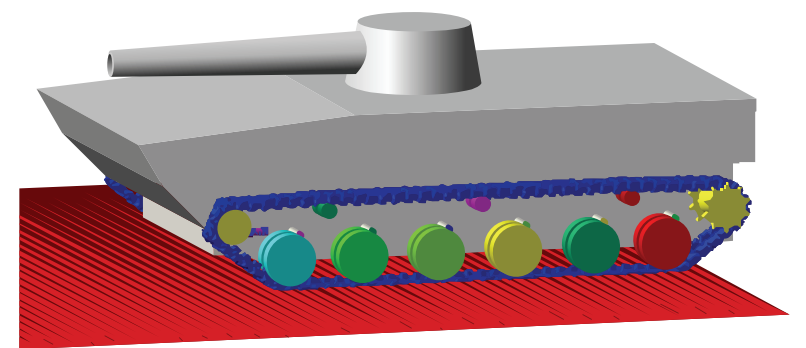

FIGURE 19: Virtual prototype model of tracked vehicle.

to the required speed, a Step function is used to drive the model:

$$
\text { Step }\left(t, t_{0}, h_{0}, x_{1}, h_{1}\right)
$$

where $t$ is the time, $s ; t_{0}$ is the initial time, $0 ; h_{0}$ is the initial function value, $0 ; x_{1}$ is the end time, $6 \mathrm{~s} ; h_{1}$ is the maximum angular velocity of the driving sprocket. The maximum speed 
TABLE 1: Main parameters of a certain type of tracked vehicle.

\begin{tabular}{lc}
\hline Parameters & Values \\
\hline Mass of half vehicle $m_{c} / \mathrm{t}$ & 20 \\
Moment of inertia $J_{c} / \mathrm{kg} \cdot \mathrm{m}^{2}$ & 115680.6 \\
Damping coefficient of suspension $c / \mathrm{kN} /(\mathrm{m} / \mathrm{s})$ & 5 \\
Spring coefficient of suspension $k / \mathrm{kN} / \mathrm{m}$ & 170 \\
Pretensioning force $F_{0} / \mathrm{kN}$ & 40 \\
Horizontal distance of road wheels' mass centers from vehicle's mass center $l_{i} / \mathrm{m}$ & {$[2.3,1.38,0.46,-0.46,-1.38,-2.3]$} \\
Vertical distance of road wheels' mass center from vehicle's mass center $h_{i} / \mathrm{m}$ & 0.3 \\
Horizontal distance of front idler's and driving sprocket's mass center from vehicle's mass center $l_{k} / \mathrm{m}$ & {$[3,-3]$} \\
Vertical distance of front idler's and driving sprocket's mass center from vehicle's mass center $h_{k} / \mathrm{m}$ & -0.1 \\
Width of a track $b / \mathrm{m}$ & 0.464 \\
Length of a track $L / \mathrm{m}$ & 0.153 \\
Radius of a road wheel $r / \mathrm{m}$ & 0.30 \\
Pitch radius of the driving sprocket $r_{p} / \mathrm{m}$ & 0.25 \\
Radius of a carrier wheel $r_{t} / \mathrm{m}$ & 0.1 \\
Radius of a front idler $r_{y} / \mathrm{m}$ & 0.2 \\
Distance between two road wheels $/ \mathrm{m}$ & 0.92
\end{tabular}

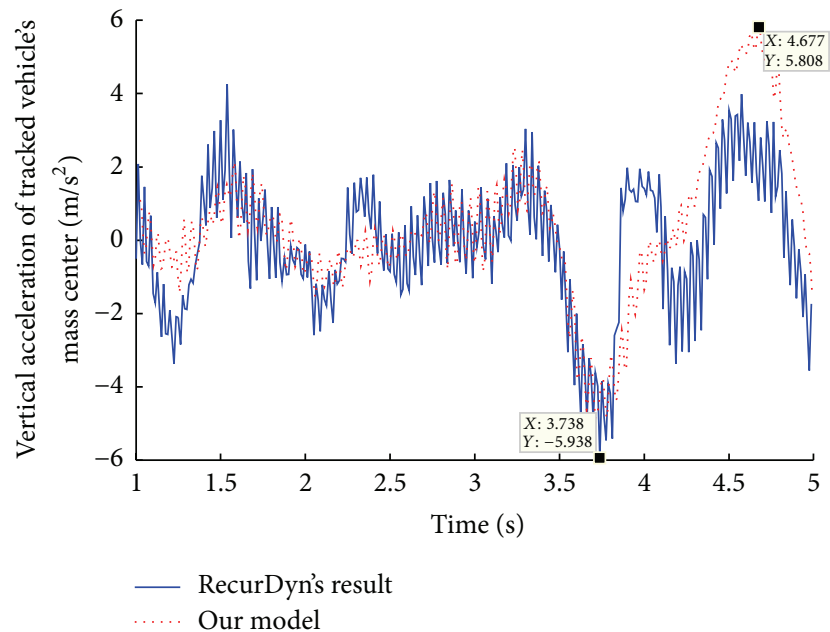

FIGURE 20: Comparison of the vertical accelerations of the vehicle body's mass center output by the model presented in this paper and RecurDyn.

of vehicle is $60 \mathrm{~km} / \mathrm{h}$, the pitch radius of driving sprocket is $0.25 \mathrm{~m}$, so its value is $67 \mathrm{rad} / \mathrm{s}$.

The vehicle model is initially static and the friction between components is neglected. When the vehicle is in a uniform rectilinear motion after 6 seconds, the vertical acceleration of the mass center of the vehicle and the tractive force of driving sprocket can be compared with the results of this paper.

It can be seen from Figure 20 that when the tracked vehicle reaches stable state, the vertical acceleration of the vehicle body's mass center calculated by the model presented in this paper is basically consistent with the result from RecurDyn, and the acceleration values are in the range of $-6 \mathrm{~m} / \mathrm{s}^{2} \sim 6 \mathrm{~m} / \mathrm{s}^{2}$, which meets the requirement that the

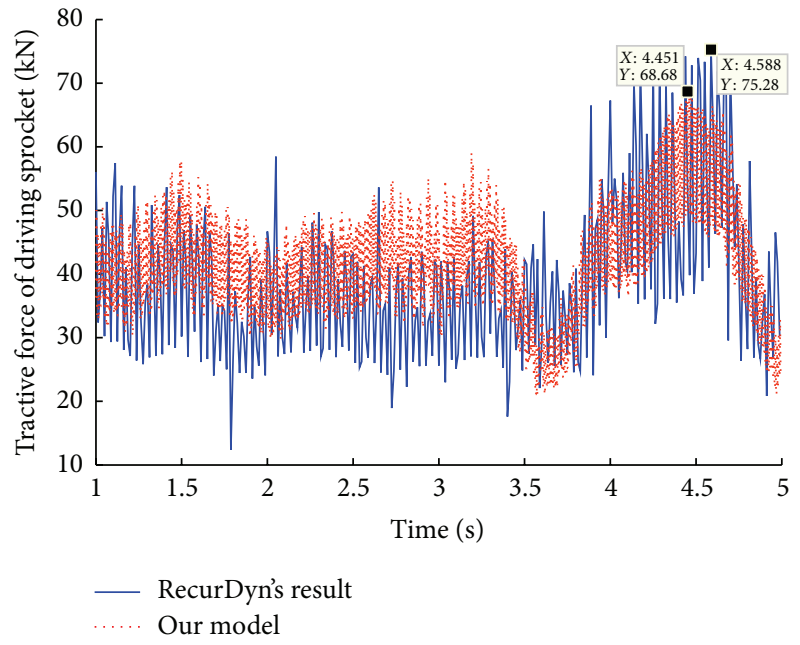

FIGURE 21: Comparison of the tractive forces output by our model and RecurDyn.

maximum vertical acceleration of tracked vehicle's mass center should be less than $1 \mathrm{~g}$. The maximum values of the model given in this paper and RecurDyn are $5.808 \mathrm{~m} / \mathrm{s}^{2}$ and $5.938 \mathrm{~m} / \mathrm{s}^{2}$, respectively. The error between them is $2.19 \%$.

It can be seen from Figure 21 that when the tracked vehicle reaches stable state, the tractive force calculated by our model is basically consistent with the result from RecurDyn if the friction among components of the vehicle and centrifugal force of the tracks are not taken into consideration. The error between our model and RecurDyn is $8.77 \%$.

The results of the model presented in this paper have some deviations from the results output by RecurDyn. This is because (1) our model is built without consideration of inertia forces of tracks and slippage of the vehicle. (2) When a road wheel passes by a track, the track will certainly rotate a 
little angle. Although the time period that a road wheel on a track is very short, neglecting this angular change may affect the result. (3) Discretization of the computation process is used to decouple the coupled problems, computation errors may be inevitably induced into the results. However, generally the computation errors are in an acceptable range, which indicates the model introduced in this paper is useful for the future optimization of design variables.

\section{Conclusion}

This paper introduces a dynamic model of tracked vehicle when the vehicle moves on the soft random terrain. The contributions of the paper include (1) the soft random terrain excitation is introduced into the model, which makes it much closer to the reality; (2) a detailed track modeling method is introduced; (3) a decoupling method is introduced to solve the coupled dynamic model. Future work would be directed towards improving the modeling of tracks by taking the inertia forces, frictions, and so forth into consideration, so that the computation errors can be further reduced.

\section{Conflict of Interests}

The authors declare that there is no conflict of interests regarding the publication of this paper.

\section{Acknowledgments}

The research work is funded by the National Natural Science Foundation of China with the Grant nos. 51175457 and 51475416. The first author is also supported by the Fundamental Research Funds for the Central Universities of China.

\section{References}

[1] Z.-D. Ma and N. C. Perkins, "A super-element of track-wheelterrain interaction for dynamic simulation of tracked vehicles," Multibody System Dynamics, vol. 15, no. 4, pp. 347-368, 2006.

[2] Z.-D. Ma and N. C. Perkins, "A track-wheel-terrain interaction model for dynamic simulation of tracked vehicles," Vehicle System Dynamics, vol. 37, no. 6, pp. 401-421, 2002.

[3] W. Y. Park, Y. C. Chang, S. S. Lee, J. H. Hong, J. G. Park, and K. S. Lee, "Prediction of the tractive performance of a flexible tracked vehicle," Journal of Terramechanics, vol. 45, no. 1-2, pp. 13-23, 2008.

[4] J. Ma, "A simplified calculation of response of a tracked vehicle simulated by road roughness," Mechanics in Engineering, vol. 34, no. 5, pp. 32-35, 2012.

[5] B. Han, X. Li, and F. Sun, "Modeling and simulation of high speed tracked vehicles," Computer Simulation, vol. 20, no. 2, pp. 107-109, 2003.

[6] W. Merhof and E. M. Hackbarth, Driving dynamics of tracked vehicle, National Defense Industry Press, Beijing, China, 1989.

[7] D. Rubinstein and R. Hitron, "A detailed multi-body model for dynamic simulation of off-road tracked vehicles," Journal of Terramechanics, vol. 41, no. 2-3, pp. 163-173, 2004.

[8] X.-G. Ma, S.-W. Pan, X.-M. You, M. Ye, and X.-L. Gong, "Mathematical models for a caterpillar driving system and its tension calculation," Journal of Vibration and Shock, vol. 33, no. 3, pp. 186-190, 2014.

[9] J. Hu, Research about the Traveling Performance of Heavy Crawler Vehicle on Soft Ground, Jilin University, Jilin, China, 2012.

[10] Y. Yang and Y. Liu, "Research process on mathematical model of road roughness," Shanghai Auto, vol. 3, pp. 23-26, 2010.

[11] J.-F. Yu, "The discussion of building road file's method in simulation of vehicle ride comfort," Technology \& Economy in Areas of Communications, vol. 6, pp. 107-109, 2010.

[12] Z. Yu, Theory of Automobile, China Machine Press, Beijing, China, 3rd edition, 2000.

[13] Y. Xu, "Computer simulation on stochastic road irregularities," Transactions of the Chinese Society of Agricultural Machinery, vol. 38, no. 1, pp. 33-36, 2007.

[14] M. G. Bekker, Off-the-Road Locomotion, University of Michigan Press, Ann Arbor, Mich, USA, 1960.

[15] M. G. Bekker, The Introduction of Ground-Vehicle Systems, China Machine Press, Beijing, China, 1978.

[16] J. Y. Wong, Theory of Ground Vehicle, Wiley, New York, NY, USA, 1978.

[17] W. Wang, Study on tracked mobile robot with manipulator in complex terrain [Dissertation], Harbin Institute of Technology, Harbin, China, 2009.

[18] Y. Cai, Research on Dynamic Performance and Transmission Type of the Engine of Bulldozer, Changan University, Xian, China, 2006.

[19] K. Zhang and S. Liu, Soil Mechanics, China Architecture \& Building Press, Beijing, China, 3rd edition, 2010.

[20] J. Janosi and B. Hanamoto, "The analytical determination of draw bar pull as a function slip for tracked vehicles in deformable soil," in Proceedings of the 1st International Conference of the International Society for Terrain-Vehicles Systems (ISTVS '61), Turin, Italy, 1961. 


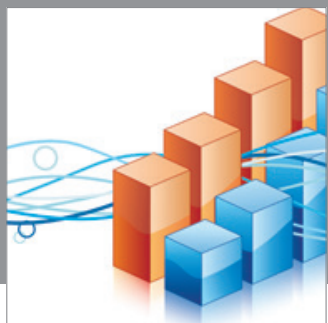

Advances in

Operations Research

mansans

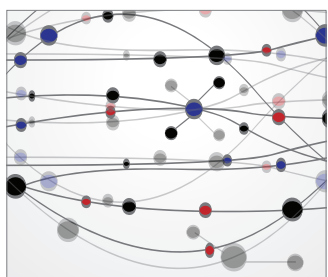

The Scientific World Journal
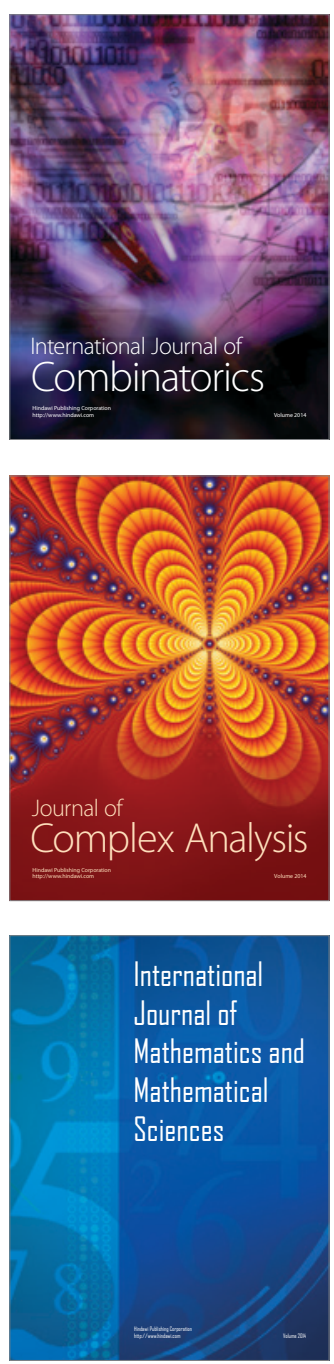
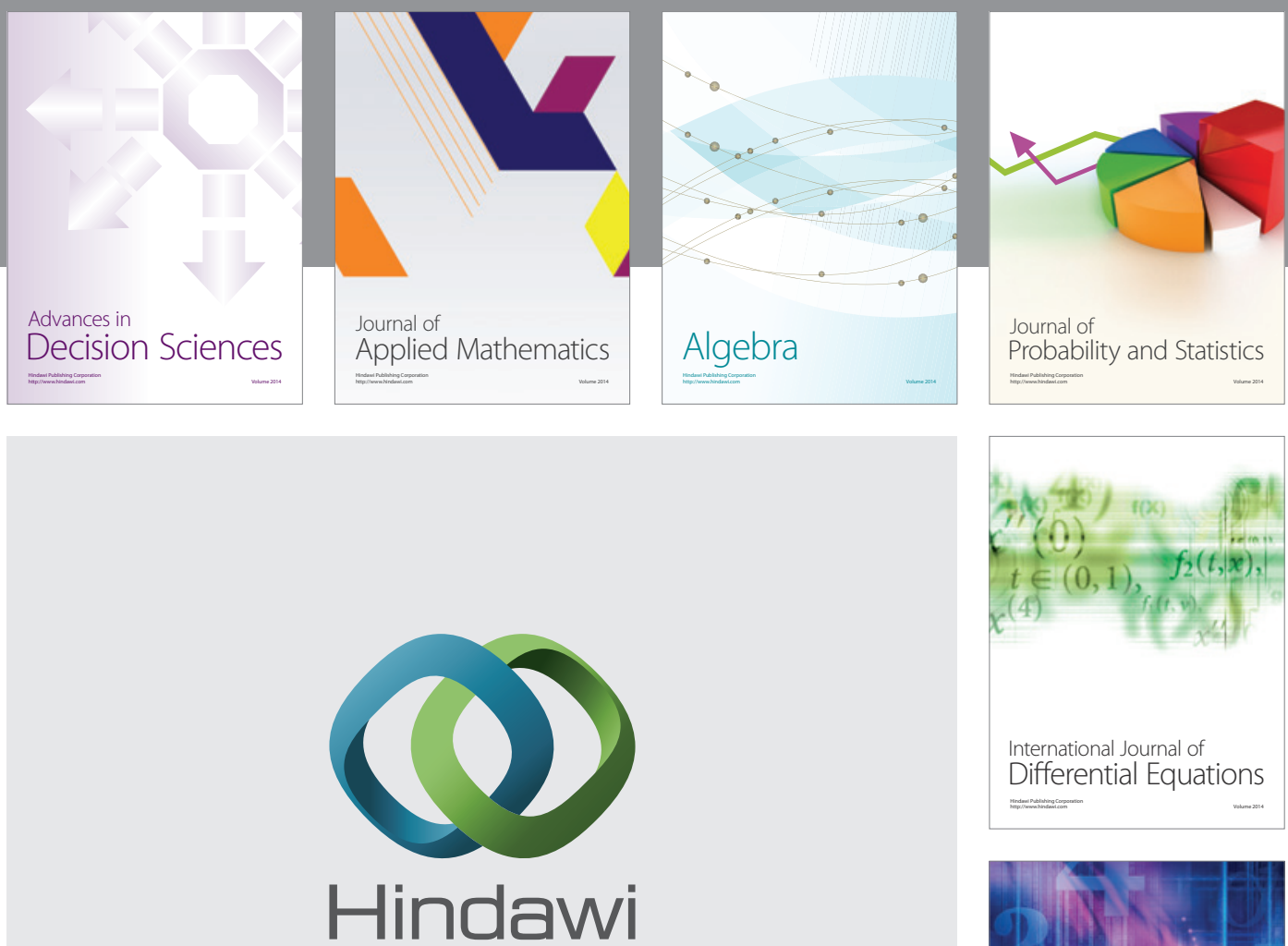

Submit your manuscripts at http://www.hindawi.com
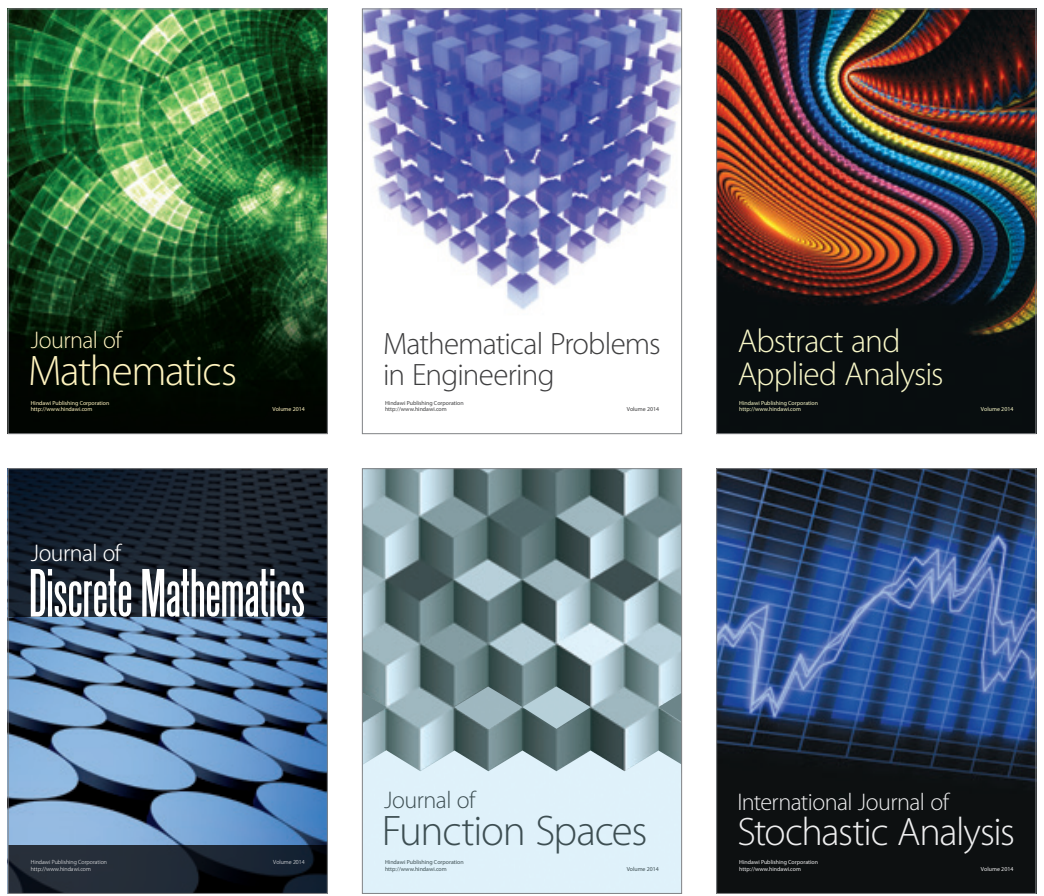

Journal of

Function Spaces

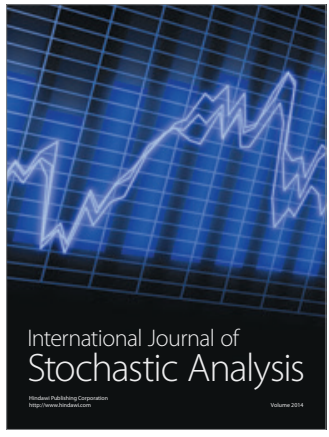

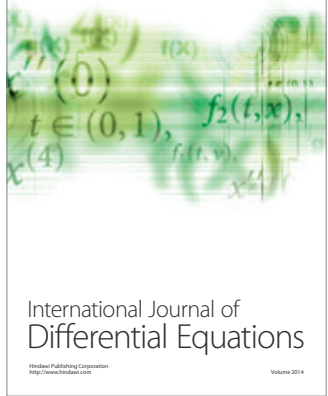
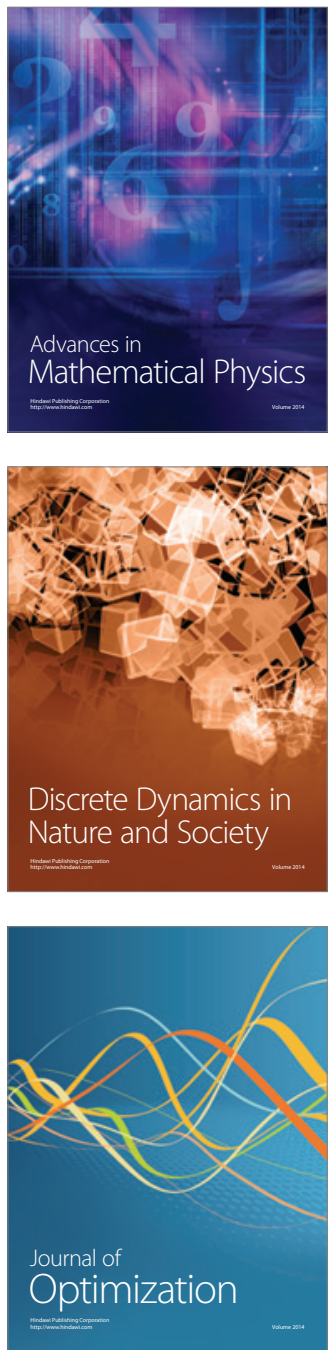